\title{
Variação radial das propriedades da madeira de Caryocar brasiliense Cambess. (pequi-do-cerrado)
}

A madeira é um dos os compostos orgânicos mais utilizados como matéria-prima no mundo. Suas características químicas, anatômicas e físicas variam sensivelmente mediante aos fatores ambientais. No Cerrado, os solos são naturalmente pobres e com estações secas prolongadas. $\mathrm{O}$ estudo das propriedades da madeira produzida nesse bioma é relevante para compreensão das adaptações morfológicas no xilema secundário para sobrevivência da planta. Objetivou-se estudar as variações e correlações entre propriedades físicas e biométricas de fibras na madeira de Caryocar brasiliense Cambess. do cerrado de Minas Gerais. Coletou-se amostras de incremento do lenho de três árvores, utilizando-se trado de Pressler na altura de 1,30 m em relação ao solo. Determinou-se a densidade, umidade e dimensões das fibras da madeira no sentido medula-casca. Os parâmetros físicos variaram em função das árvores e da posição radial. As médias de densidade básica e umidade verde foram de $0,56 \mathrm{~g} / \mathrm{cm}^{3}$ e $96,28 \%$, respectivamente. A tendência observada nas propriedades físicas repetiu-se para os parâmetros biométricos das fibras. As médias de largura, espessura e comprimento de fibras foram $24,13 \mu \mathrm{m}, 5,16 \mu \mathrm{m}$ e $2,65 \mathrm{~mm}$, respectivamente. 0 padrão de variação radial da madeira de pequi-do-cerrado indicou que ao se afastar da medula, a densidade básica e o comprimento das fibras são crescentes. Por outro lado, a umidade verde é decrescente. Para largura e espessura da parede celular das fibras não se detectou tendências claras. Entre as árvores de C. brasiliense, constatouse variação e correlação das propriedades físicas e particularidades anatômicas da madeira na mesma tipologia de cerrado.

Palavras-chave: Cerrado; Xilema; Anatomia da madeira; Física da madeira; Biometria de fibras.

\section{Radial profile in wood properties of Caryocar brasiliense Cambess. (pequi-do-cerrado)}

\begin{abstract}
Wood is one of the most widely used organic compounds as a raw material in the world. Its chemical, anatomical and physical characteristics vary significantly due to environmental factors. In the Cerrado, the soils are naturally poor and have prolonged dry seasons. The study of the properties of the wood produced in this biome is relevant for understanding the morphological and anatomical adaptations in the xylem for plant survival. The objective was to study the variations and correlations between physical properties and fiber biometry in the wood of Caryocar brasiliense Cambess. of the cerrado of Minas Gerais. Core of wood increment were collected from three trees, using Pressler's borer at a height of $1.30 \mathrm{~m}$ in relation to the soil. The density, moisture and biometry of the wood fibers in the pith-bark direction were determined. The physical parameters varied according to the trees and the radial position. The averages of basic density and green humidity were $0.56 \mathrm{~g} / \mathrm{cm}^{3}$ and $96.28 \%$, respectively. The trend observed in physical properties was repeated for the biometric parameters of the fibers. The mean width, wall thickness and length of fibers were $24.13 \mu \mathrm{m}, 5.16 \mu \mathrm{m}$ and $2.65 \mathrm{~mm}$, respectively. The radial variation pattern of pequi-do-cerrado wood indicated that when moving away from the spinal cord, the basic density and length of the fibers are increasing. On the other hand, green humidity is decreasing. For the width and thickness of the cell wall of the fibers, no clear trends were detected. Among the trees of $\mathrm{C}$. brasiliense there was variation and correlation of the physical properties and anatomical characteristics of the wood in the same type of cerrado.
\end{abstract}

Keywords: Cerrado; Xylem; Wood anatomy; Wood physics; Fiber biometry.

Topic: Ciências Florestais

Reviewed anonymously in the process of blind peer.
Received: $21 / 02 / 2020$

Approved: 09/03/2020
Christovão Pereira Abrahão (iD)

Universidade Federal dos Vales do Jequitinhonha e Mucuri, Brasil http://lattes.cnpq.br/3470385452325199

http://orcid.org/0000-0003-4329-9308

cabrahao@gmail.com

Ivo Perceu Ferreira Campos (iD)

Universidade Federal dos Vales do Jequitinhonha e Mucuri, Brasil http://lattes.cnpq.br/4025717560663640

http://orcid.org/0000-0002-7416-7351

ivoperceu@hotmail.com

Cilene Cristina Borges (iD)

Universidade Tecnológica Federal do Paraná, Brasil

http://lattes.cnpq.br/3308819681621127

http://orcid.org/0000-0002-7416-7351

cilene@utfpr.edu.br

Thiago Moreira Cruz
Universidade Federal de Lavras, Brasil
http://lattes.cnpq.br/7811942414257946
$\begin{aligned} & \text { http://orcid.org/0000-0003-2808-1810 } \\ & \text { thiago.moreira.cruz@gmail.com }\end{aligned}$
Adriano Reis Prazeres Mascarenhas 10
Universidade Federal de Lavras, Brasil
http://lattes.cnpq.br/7050276331020244
http://orcid.org/0000-0002-7554-3590
adriano.mascarenhas@unir.br
Caik Tonelli Elisio Faria (D)
Universidade Federal de Lavras, Brasil
http://lattes.cnpq.br/0919865797988547
http://orcid.org/0000-0002-0138-6801
caiktonelli@gmail.com

Paulo Junio Duarte (iD)

Universidade Federal de Lavras, Brasil http://lattes.cnpq.br/1283844646664824 http://orcid.org/0000-0002-7319-3101

pauloduarte.floresta@gmail.com
Referencing this:

ABRAHÃO, C. P.; CAMPOS, I. P. F.; BORGES, C. C.; CRUZ, T. M. MASCARENHAS, A. R. P.; FARIA, C. T. E.; DUARTE, P. J.. Variação radial das propriedades da madeira de Caryocar brasiliense Cambess. (pequido-cerrado). Revista Ibero Americana de Ciências Ambientais, v.11, n.2, p.14-22, 2020. DOI: http://doi.org/10.6008/CBPC21796858.2020 .002 .0002 


\section{INTRODUÇÃO}

Dentre os materiais de origem biológica disponíveis na natureza a madeira tem grande destaque em diversas aplicações ao redor do mundo. Por apresentar alta resistência em relação a massa, versatilidade, biodegradabilidade é um material renovável interessante do ponto de vista econômico e ambiental (KIM et al., 2016). O uso da madeira é um mecanismo alternativo para o sequestro e armazenamento de carbono, contribuindo para redução de $\mathrm{CO}_{2}$ atmosférico (FPL, 2010).

A madeira é formada na região do câmbio vascular e desempenha funções vitais para a árvore, principalmente para transporte e armazenamento de água bem como de suporte mecânico arbóreo, funções essas executadas por diferentes células no xilema, com grandes alterações nas estruturas do tecido entre espécies e dentro da árvore nos sentidos axial e radial (RAVEN et al., 2014). Devido a isso, a estrutura do lenho é sensivelmente afetada pelos fatores intrínsecos e extrínsecos ao organismo da árvore. 0 entendimento dos mecanismos envolvidos nesse fenômeno é o principal desafio dos pesquisadores, pois torna-se necessário correlacionar a plasticidade, a adaptação, fatores genéticos e ambientais que influenciam na xilôgenese.

O estudo da anatomia da madeira é uma opção para atender esse objetivo, uma vez que permite integrar as relações hídricas entre planta e solo e os mecanismos fisiológicos das células, os quais configuram a disposição e morfologia dos elementos celulares ao longo do fuste para a sobrevivência da planta, afetando diretamente as propriedades tecnológicas da madeira (BATTIPAGLIA et al., 2014).

Entre as características tecnológicas, a densidade da madeira é relatada por muitos pesquisadores como principal traço funcional ligado ao histórico de vida da planta (MULLER-LANDAU, 2004; CHAVE et al., 2009; WILLIAMSON et al., 2011). Essa propriedade física tem sido crucial nos programas de contabilidade de carbono em florestas tropicais (CHAVE et al., 2009), uma vez que é empregada no cálculo da biomassa seca a partir dos resultados de inventários volumétricos.

Além de suas consequências amplamente reconhecidas pelas correlações com as propriedades mecânicas para a sua utilização adequada, a densidade da madeira é também determinante para a eficiência no transporte de água do xilema e das relações hídricas de toda a planta (MEINZER, 2003). Por efeito disso, entender os processos biológicos que permitem às árvores modificar o desenvolvimento da madeira para mitigar os efeitos adversos da seca é crucial para a criação de estratégias bem-sucedidas para o futuro do manejo e conservação das florestas (RODRIGUEZ-ZACCARO et al., 2019).

Em savanas do tipo Cerrado Stricto Sensu, algumas espécies como o pequi-do-cerrado, Caryocar brasiliense Cambess. (Caryocaraceae), demonstram ótimas habilidades para sobrevivência e estão distribuídas por todo o Cerrado Brasileiro. Essa espécie pode atingir mais de dez metros de altura e seis metros de largura de copa (SILVA JÚNIOR, 2012). Devido ao seu uso na culinária tradicional e produção do óleo essencial, o pequi é um recurso com potencial comercial e de importância econômica para as regiões Centro-Oeste e Sudeste do Brasil (ARAÚJO, 1995; LEITE et al., 2006; MOURA et al., 2013).

Objetivou-se investigar as variações e correlações entre as propriedades físicas e características das 
fibras na madeira de C. brasiliense, provenientes do cerrado de Minas Gerais.

\section{MATERIAIS E MÉTODOS}

\section{Caracterização da região de estudo}

O material utilizado nesse trabalho foi obtido na Fazenda Experimental do Moura, pertencente a Universidade Federal dos Vales do Jequitinhonha e Mucuri (UFVJM), localizada no município de Curvelo, Minas Gerais (Altitude de 633 m entre a latitude 1849'25,79"S e longitude 4424'13,32"O) (Figura 1).

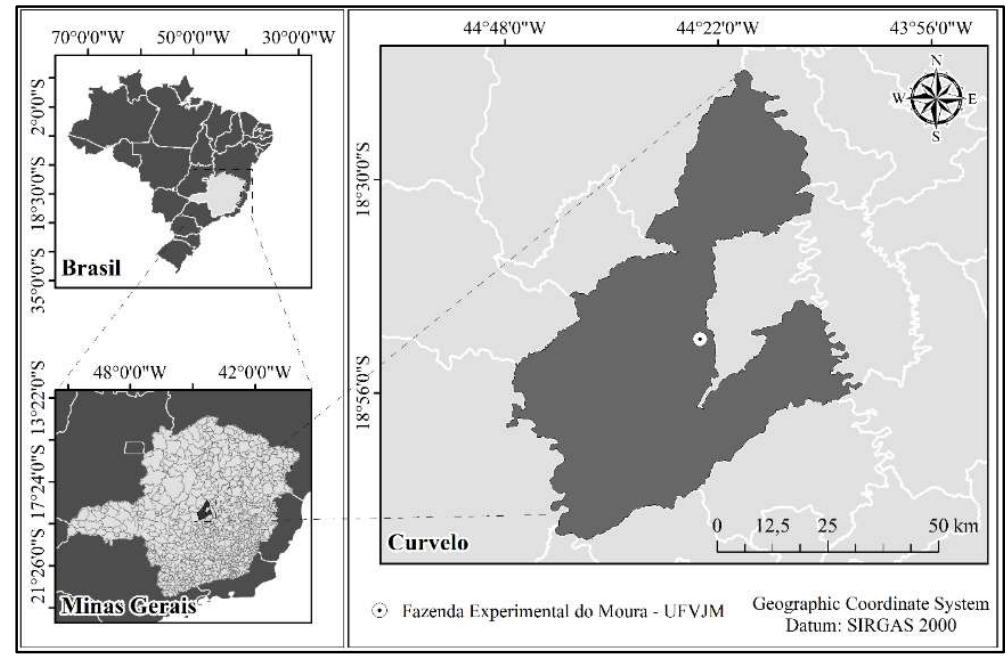

Figura 1: Localização da área de estudo, Fazenda Experimental do Moura, Curvelo, Minas Gerais, Brasil.

A vegetação predominante é típica de Cerrado Stricto Sensu. O solo da região é o latossolo vermelho distrófico (SANTOS et al., 2018). O clima é do tipo Cwa, predominantemente seco, com temperaturas médias em torno de $28^{\circ} \mathrm{C}$ e precipitação média em torno de $1308 \mathrm{~mm}$ por ano (ALVARES et al., 2013).

\section{Seleção de árvores e amostragem de madeira}

Atendendo-se ao mínimo estabelecido na norma ASTM D 5536 (ASTM, 2017), foram selecionadas três árvores de Caryocar brasiliense, as quais foram identificadas com os códigos 1, 2 e 3. Além disso, mediuse os DAP das árvores, que foram em torno de $20,83 \mathrm{~cm} \pm 4,53 \mathrm{~cm}$. Posteriormente, em cada árvore foram extraídas duas amostras (baguetas) utilizando-se trado de Pressler (Increment borer), com 3 hélices e broca com $5 \mathrm{~mm}$ de diâmetro interno e $40 \mathrm{~mm}$ de comprimento.

Para isso, introduziu-se o trado na altura de 1,30 m (DAP), perpendicularmente ao eixo da árvore, objetivando-se penetrar todo diâmetro do lenho passando pela medula. As baguetas extraídas, ainda em condição de saturação de fibras, foram seccionadas paralelamente às fibras em diferentes posições radiais, resultando em dezenas de corpos de prova com, aproximadamente, $2 \times 5 \mathrm{~mm}$ (comprimento $\times$ diâmetro).

Enumerou-se os corpos de prova segundo sua posição em relação à medula, em seguida o material foi destinado à determinação da densidade, teor de umidade verde e biometria das fibras (Figura 2). 0 seccionamento foi realizado com o auxílio de estilete de aço, pressionando-o firmemente no sentido das fibras. 


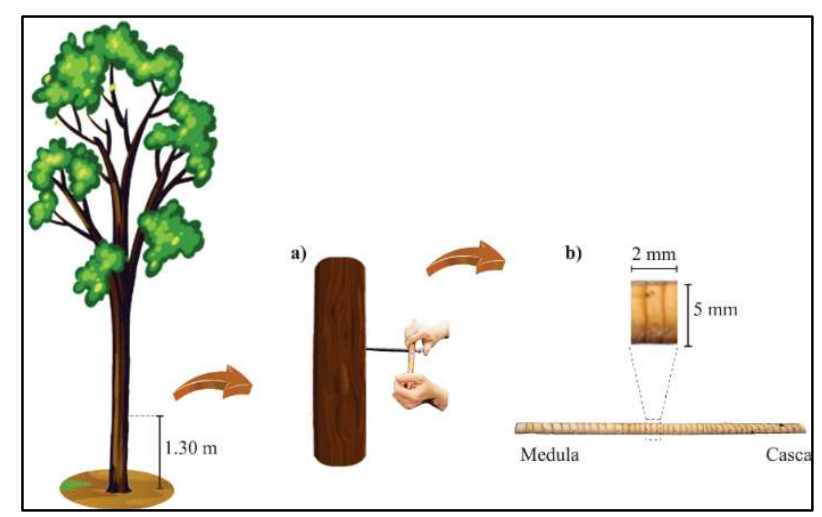

Figura 2: Esquema de extração das amostras. a) Aplicação do trado Pressler na região do DAP (1,30 m) para retirada das baguetas. b) Retirada de filamento da bagueta entre a região da medula e casca para obtenção dos parâmetros tecnológicos.

\section{Determinação da umidade e densidade básica}

Determinou-se a massa e as dimensões (diâmetro e altura do material saturado) dos corpos de prova oriundas das baguetas, com o auxílio de uma balança analítica e micrômetro, respectivamente. Para isso, as amostras foram submetidas à secagem ao ar até observação de massa constante. Posteriormente, conduziuse o material para estufa com circulação forçada de ar em temperatura de $105^{\circ} \mathrm{C} \pm 5^{\circ} \mathrm{C}$, para obtenção da massa anidra. De posse dos valores de massa úmida, massa seca e volume saturado das amostras, calculouse a umidade verde (Eq. 1) e a densidade básica da madeira (Eq. 2), em diferentes regiões desde a medula até a casca.

$$
\begin{gathered}
\mathrm{Uv}=\frac{(\mathrm{Mu}-\mathrm{Ms})}{\mathrm{Ms}} \times 100 \\
\mathrm{Db}=\frac{\mathrm{Ms}}{\mathrm{Vsat}}
\end{gathered}
$$

Em que: $\mathrm{Uv}=$ teor de umidade da madeira verde (\%); $\mathrm{Mu}=$ massa da amostra de madeira úmida (g) Ms = massa da amostra seca a $0 \%$ de umidade $(\mathrm{g}) ; \mathrm{Db}=$ densidade básica $\left(\mathrm{g} / \mathrm{cm}^{3}\right) ; \mathrm{e}$ Vsat = volume da amostra de madeira saturada $\left(\mathrm{cm}^{3}\right)$.

\section{Biometria das fibras}

Após serem pesadas à seco, as amostras foram novamente saturadas e submetidas ao processo de maceração proposto por Franklin (1945). Dessa forma, os retalhos de madeira foram imersos em ácido acético glacial e peróxido de hidrogênio $(1: 1 \mathrm{v} / \mathrm{v})$, à temperatura de $60^{\circ} \mathrm{C}$ por 24 horas. Após o processo de maceração as fibras foram coradas com solução alcoólica de safranina a 0,5\%, tendo sido utilizadas 20 fibras em cada amostra para determinação das dimensões das fibras. A largura das fibras e espessura das paredes celular foram obtidas através de imagens do macerado com o auxílio de um microscópio óptico, no aumento de 40 vezes. O comprimento das fibras foi determinado por meio de microscópio estereoscópico, com aumento de 30 vezes.

\section{Análises estatísticas}

As análises estatísticas foram realizadas no software R (R CORE TEAM, 2017), empregando-se o nível 
de significância $(p<0,05)$. Para a comparação das médias dos valores obtidos, utilizou-se o teste t de Student. A correlação entre as propriedades da madeira aqui avaliadas foi determinada pela matriz de correlação linear de Pearson $(p<0,05)$.

\section{RESULTADOS E DISCUSSÃO}

\section{Densidade e umidade verde}

Houveram diferenças para densidade básica entre as árvores analisadas, sendo observado na árvore 2 o menor valor para esse parâmetro (Tabela 1), no entanto, ao se observar o desvio padrão, a variação da densidade em cada árvore foi muito baixa. Quanto a umidade, o comportamento observado foi oposto, isso porque a densidade é inversamente proporcional à porosidade da madeira, ou seja, quanto menor quantidade de espaços porosos menor será o armazenamento de água livre nas cavidades celulares (MELO et al., 2013), como pode ser visto nas árvores 1 e 3.

Tabela 1: Resumo estatístico para densidade básica (Db) e umidade verde (Uv) da madeira de Caryocar brasiliense (pequi-do-cerrado).

\begin{tabular}{|l|l|l|l|l|}
\hline Parâmetros & Valores & Árvore 1 & Árvore 2 & Árvore 3 \\
\hline \multirow{4}{*}{$\mathrm{Db}\left(\mathrm{g} / \mathrm{cm}^{3}\right)$} & Mínimo & 0,45 & 0,35 & 0,43 \\
\cline { 2 - 5 } & Média & $0,58 \mathrm{a}$ & $0,52 \mathrm{~b}$ & $0,59 \mathrm{a}$ \\
\cline { 2 - 5 } & Máximo & 0,75 & 0,68 & 0,78 \\
\cline { 2 - 5 } & Desvio padrão & 0,06 & 0,08 & 0,08 \\
\hline \multirow{4}{*}{ Uv (\%) } & Mínimo & 46,88 & 64,29 & 53,57 \\
\cline { 2 - 5 } & Média & $90,64 \mathrm{~b}$ & $102,50 \mathrm{a}$ & $95,40 \mathrm{ab}$ \\
\cline { 2 - 5 } & Máximo & 139,13 & 146,15 & 126,19 \\
\cline { 2 - 5 } & Desvio padrão & 23,78 & 20,99 & 18,45 \\
\hline
\end{tabular}

Médias na mesma linha, seguidas pela mesma letra, não diferem entre si pelo teste de $t$ a $5 \%$ de probabilidade.

A seguir na tabela 2, verifica-se que não houveram diferenças entre as árvores 2 e 3 para comprimento e espessura de fibras. Nas árvores 1 e 2 não foram observadas diferenças entre as médias de largura de fibras, nas quais se obteve os menores valores para esse parâmetro. Assim como para densidade, o desvio padrão em cada árvore não foi elevado.

Tabela 2: Resumo estatístico para biometria de fibras da madeira de Caryocar brasiliense (pequi-do-cerrado).

\begin{tabular}{|c|c|c|c|c|}
\hline Parâmetros & Valores & Árvore 1 & Árvore 2 & Árvore 3 \\
\hline \multirow[t]{4}{*}{ Comprimento $(\mathrm{mm})$} & Mínimo & 1,66 & 2,66 & 2,33 \\
\hline & Média & $2,27 c$ & $3,00 a$ & $2,70 \mathrm{~b}$ \\
\hline & Máximo & 3,43 & 3,41 & 3,01 \\
\hline & Desvio padrão & 0,30 & 0,20 & 0,18 \\
\hline \multirow[t]{4}{*}{ Largura $(\mu \mathrm{m})$} & Mínimo & 16,33 & 18,13 & 21,63 \\
\hline & Média & $23,63 \mathrm{~b}$ & $23,65 b$ & $26,02 a$ \\
\hline & Máximo & 29,36 & 28,57 & 31,78 \\
\hline & Desvio padrão & 3,38 & 2,39 & 2,68 \\
\hline \multirow[t]{4}{*}{ Espessura $(\mu \mathrm{m})$} & Mínimo & 4,48 & 4,40 & 4,63 \\
\hline & Média & $5,86 a$ & $5,40 b$ & $5,54 a b$ \\
\hline & Máximo & 8,39 & 6,54 & 6,79 \\
\hline & Desvio padrão & 0,90 & 0,51 & 0,54 \\
\hline
\end{tabular}

Médias na mesma linha, seguidas pela mesma letra, não diferem entre si pelo teste de $\mathrm{t}$ a $5 \%$ de probabilidade.

Em geral, as variações da densidade da madeira dependem das mudanças na proporção das células do xilema secundário e da espessura da parede celular da fibra de sustentação. O que sugere que cada árvore 
de pequi-do-cerrado tem um histórico de vida e uma estratégia diferente de sobrevivência. A variabilidade na densidade da madeira exemplifica a natureza altamente plástica do crescimento e da forma das plantas.

Valente et al. (2013) observaram que na direção radial, a variação das propriedades da madeira de Anadenanthera peregrina (L.) Speg. (Fabaceae) foram significativas, sendo que a densidade básica e aparente correlacionaram-se com as propriedades físico-mecânicas e características anatômicas avaliadas. Segundo Parolin (2002) o aumento da densidade básica da madeira está associado com as estratégias de crescimento da árvore em seu ambiente. A complexidade de respostas fotossintéticas e de relações hídricas nas espécies vegetais do Cerrado está relacionada com o clima e o solo de tal ambiente (PALHARES et al., 2010).

Para Meinzer (2003), a estreita relação entre a densidade da madeira e o histórico de vida da planta, reflete o fato de que o xilema secundário desempenha papel fisiológico fundamental no transporte da seiva através dos poros e de suporte mecânico através das fibras na sustentação do corpo e resistência à flexão da planta. No sentido radial, este fato é confirmado pelos coeficientes de correlação positivos e significativos entre propriedades físicas e a biometria das fibras do xilema. Diferenças significativas e correlações foram observadas entre indivíduos e posições na madeira entre a densidade básica, a umidade com o comprimento das fibras (Tabela 3).

Tabela 3: Matriz de correlação entre as variáveis estudadas, dentro da mesma árvore e agrupando-se as três árvores de Caryocar brasiliense (pequi-do-cerrado).

\begin{tabular}{|c|c|c|c|c|c|c|c|}
\hline Árvore & Variáveis & Posição & $\mathrm{Db}$ & Uv & Largura & Espessura & Comprimento \\
\hline Geral & \multirow[t]{4}{*}{ Posição } & - & $0,5^{*}$ & $-0,7^{*}$ & 0,1 & $-0,2$ & $0,4^{*}$ \\
\hline 1 & & - & $0,6^{*}$ & $-0,9 *$ & $0,3^{*}$ & $-0,1$ & $0,8^{*}$ \\
\hline 2 & & - & $0,6^{*}$ & $-0,7^{*}$ & $0,3^{*}$ & $-0,2$ & $0,6^{*}$ \\
\hline 3 & & - & $0,7^{*}$ & $-0,7^{*}$ & $-0,5^{*}$ & $-0,6^{*}$ & $0,4^{*}$ \\
\hline Geral & \multirow[t]{4}{*}{$\mathrm{Db}$} & $0,5^{*}$ & - & $-0,7^{*}$ & 0,0 & $-0,1$ & $-0,1$ \\
\hline 1 & & $0,6^{*}$ & - & $-0,6^{*}$ & $-0,1$ & $-0,2$ & 0,3 \\
\hline 2 & & $0,6^{*}$ & - & $-0,7^{*}$ & 0,2 & $-0,1$ & $0,4^{*}$ \\
\hline 3 & & $0,7^{*}$ & - & $-0,9 *$ & $-0,5^{*}$ & $-0,4^{*}$ & $0,4^{*}$ \\
\hline Geral & \multirow[t]{4}{*}{ Uv } & $-0,7^{*}$ & $-0,7^{*}$ & - & $-0,1$ & 0,0 & $-0,1$ \\
\hline 1 & & $-0,9 *$ & $-0,6^{*}$ & - & $-0,3^{*}$ & 0,0 & $-0,6^{*}$ \\
\hline 2 & & $-0,7^{*}$ & $-0,7^{*}$ & - & $-0,2$ & 0,1 & $-0,4^{*}$ \\
\hline 3 & & $-0,7^{*}$ & $-0,9 *$ & - & $0,5^{*}$ & $0,5^{*}$ & $-0,4^{*}$ \\
\hline Geral & \multirow[t]{4}{*}{ Largura } & 0,1 & 0,0 & $-0,1$ & - & $0,6^{*}$ & 0,1 \\
\hline 1 & & $0,3^{*}$ & $-0,1$ & $-0,3^{*}$ & - & $0,7^{*}$ & 0,2 \\
\hline 2 & & $0,3^{*}$ & 0,2 & $-0,2$ & - & $0,5^{*}$ & 0,2 \\
\hline 3 & & $-0,5^{*}$ & $-0,5^{*}$ & $0,5^{*}$ & - & $0,5^{*}$ & $-0,4^{*}$ \\
\hline Geral & \multirow[t]{4}{*}{ Espessura } & $-0,2$ & $-0,1$ & 0,0 & $0,6^{*}$ & - & $-0,3^{*}$ \\
\hline 1 & & $-0,1$ & $-0,2$ & 0,0 & $0,7^{*}$ & - & $-0,1$ \\
\hline 2 & & $-0,2$ & $-0,1$ & 0,1 & $0,5^{*}$ & - & 0,0 \\
\hline 3 & & $-0,6^{*}$ & $-0,4^{*}$ & $0,5^{*}$ & $0,5^{*}$ & - & $-0,4$ \\
\hline Geral & \multirow[t]{4}{*}{ Comprimento } & $0,4^{*}$ & $-0,1$ & $-0,1$ & 0,1 & $-0,3^{*}$ & - \\
\hline 1 & & $0,8^{*}$ & 0,3 & $-0,6 *$ & 0,2 & $-0,1$ & - \\
\hline 2 & & $0,6^{*}$ & $0,4^{*}$ & $-0,4^{*}$ & 0,2 & 0,0 & - \\
\hline 3 & & $0,4^{*}$ & $0,4^{*}$ & $-0,4^{*}$ & $-0,4^{*}$ & $-0,4$ & - \\
\hline
\end{tabular}

Legenda: *= correlação significativa a $5 \%$ de probabilidade.

Na Figura 3, visualiza-se o comportamento da densidade básica e o comprimento de fibras com valores correlacionados positivamente do distanciamento da medula para a região do alburno da árvore $1 \mathrm{e}$ 2, a árvore 3 não demonstrou tendência clara. Observou-se valores positivos para as 3 árvores entre o comprimento da fibra vegetal e a densidade básica, porém, apenas as árvores 2 e 3 apresentaram valores significativos estatisticamente $(\alpha=5 \%)$. Geralmente, as fibras se desenvolvem no comprimento à medida que 
a árvore cresce em diâmetro, até atingir um patamar a partir do qual, varia pouco nesta característica, o que indica que esta propriedade anatômica pode auxiliar na avaliação do grau de maturidade do lenho (PANSHIN et al., 1980).

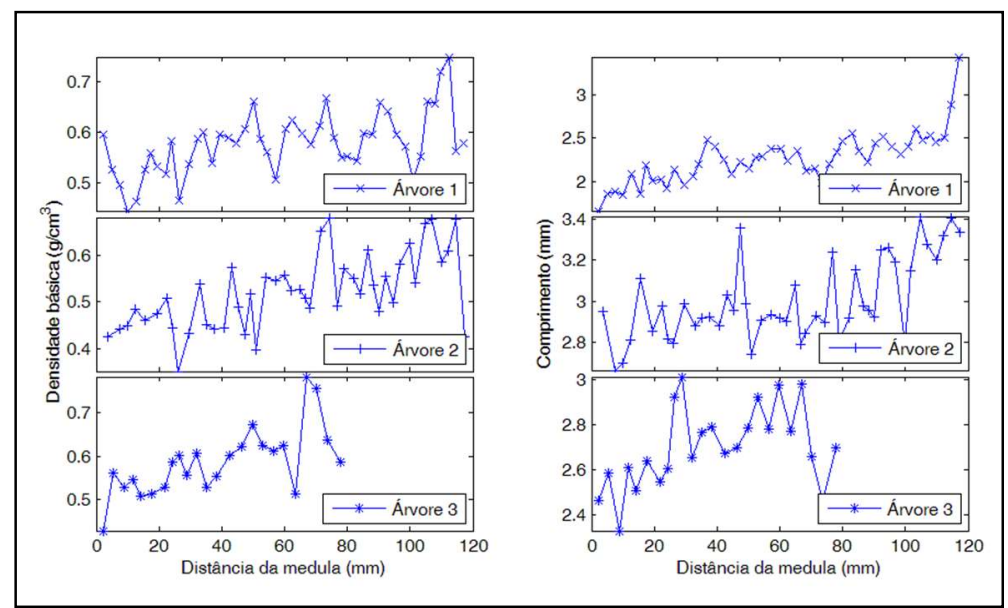

Figura 3: Variação radial dos valores médios para a densidade básica e para o comprimento das fibras da madeira de Caryocar brasiliense (pequi-do-cerrado).

Além do comprimento de fibras, constatou-se que a variação observada em espessura e largura das fibras, influenciou nas propriedades físicas da madeira entre os indivíduos de $C$. brasiliense em uma mesma tipologia de cerrado. A morfologia das fibras de Caryocar brasiliense demonstrou diferentes comportamentos, a espessura da parede celular apresentou correlação negativa com a densidade básica na posição medula-casca, mas, somente a árvore 3 exibiu um coeficiente significativo. Quanto à largura das fibras, a árvore 3 obteve média superior significativa de 26,02 $\mu \mathrm{m}$, comparado aos 23,63 $\mu \mathrm{m}$ da amostra 1 e 23,65 $\mu \mathrm{m}$ da amostra 2, esses valores correlacionaram-se positivamente com a espessura da parede celular (Tabela 2).

A largura das fibras das árvores 1, 2, e 3 correlacionaram positivamente com a espessura da parede celular. A espessura da parede celular apresentou correlação negativa com posição medula-casca, porém apenas a árvore 3 exibiu um coeficiente significativo; agrupando-se todas as árvores, a correlação é negativa e significativa (Tabela 2). Fazendo uma análise da figura 4, para as árvores 1, 2 não se observa de forma clara alguma tendência no sentido medula casca.

Segundo Valente et al. (2013) e Longui et al. (2010), para as variações medula-casca da espessura da parede, são observados diferentes padrões em espécies nativas do Brasil. Voigt et al. (2010), analisando o lenho de pequi em duas fitofisionomias de Cerrado, encontraram valores médios entre 2 e 2,15 mm para comprimento de fibras, para a largura observaram de 23,6 a $24 \mu \mathrm{m}$ e para espessura da parede, valores em torno de $10 \mu \mathrm{m}$. Em comparação com nossos dados, estes se assemelham apenas para a largura da célula. Os comprimentos de fibras de pequi encontrados aqui, variaram de $2,27 \mathrm{~mm}$ a $3 \mathrm{~mm}$ e são considerados grandes em comparação com fibras de clones comerciais de eucalipto que atingem pouco mais de $1 \mathrm{~mm}$ (EVANGELISTA et al., 2010; RAMOS et al., 2011; MAURI et al., 2017).

Algumas aparentes inconsistências para as correlações entre a largura das fibras e a posição medulacasca puderam ser detectadas neste trabalho, sendo positiva para as árvores 1 e 2, e negativa para a árvore 
3. Neste trabalho, as árvores foram selecionadas aleatoriamente de acordo com a disponibilidade do ambiente nativo, as diferenças entre locais dentro de uma mesma fitofisionomia e as influências ambientais possivelmente alteraram o grau de maturidade das 3 madeiras analisadas.

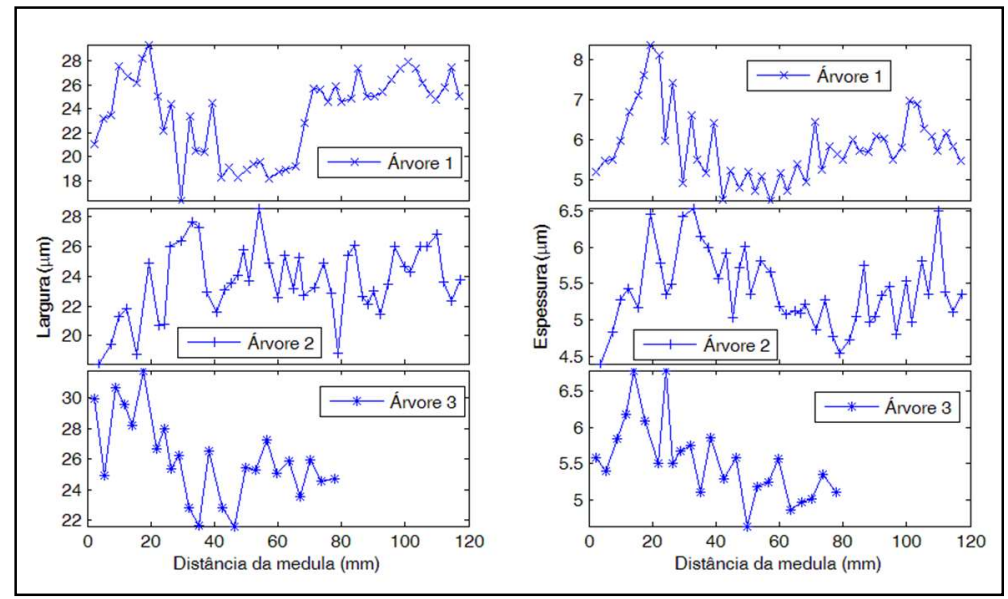

Figura 4: Variação radial dos valores médios para largura e espessura da parede celular de fibras da madeira de Caryocar brasiliense (pequi-do-cerrado).

De maneira geral, os indivíduos de $C$. brasiliense em uma mesma tipologia de Cerrado variaram a morfologia das fibras e consequentemente as suas propriedades físicas. Os valores biométricos das células de sustentação, a densidade básica e a umidade da madeira foram significativas, comprovando que cada árvore tem um histórico de vida e uma estratégia diferente de sobrevivência. As flutuações observadas para os valores das propriedades ao longo do perfil radial podem estar relacionadas às condições do sítio e disponibilidade hídrica em que as árvores de Cerrado foram submetidas durante seu desenvolvimento. Portanto, entender a evolução e os padrões espaciais na arquitetura da planta e a sua relação com os atributos tecnológicos como a densidade e teor de umidade tem grande importância para a compreensão dos processos ecológicos e fisiológicos em árvores tropicais.

\section{CONCLUSÕES}

Constatou-se variação nas propriedades físicas e características anatômicas da madeira de $C$. brasiliense na mesma tipologia de cerrado. Houve correlação positiva no sentido medula-casca entre densidade básica e comprimento das fibras. A umidade e espessura da parede celular das fibras apresentaram correlação negativa na direção da casca. Por meio do estudo integrado de propriedades da madeira e variáveis ambientais, é possível obter informações para estimar idade das árvores, determinar a transição entre lenho juvenil e adulto e predizer o comportamento tecnológico da madeira.

\section{REFERÊNCIAS}

ALVARES, C. A.; STAPE, J. L.; SENTELHAS, P. C.; MORAES GONÇALVES, J. L.; SPAVOREK, G.. Köppen's climate classification map for Brazil. Meteorologische Zeitschrift, v.22, n.6, p.711-728, 2013. DOI:

https://doi.org/10.1127/0941-2948/2013/0507

ARAÚJO, F. D.. A review of Caryocar brasiliense (Caryocaraceae) - an economically valuable species of the central Brazilian cerrado. Economic Botany, v.49, n.1, p.4048, 1995. DOI: https://doi.org/10.1007/BF02862276

ASTM. American Society for Testing and Materials. D 5536 Standard practice for sampling forest trees for determination of clear wood properties. West Conshohocken: ASTM, 2017. 
BATTIPAGLIA, G.; MICCO; V.; SASS-KLAASSEN; TOGNETTI, R.; MÄKELA, A.. WSE symposium: Wood growth under environmental changes: the need for a multidisciplinary approach. Tree physiology, v.34, n.8, p.787-791, 2014. DOI: https://doi.org/10.1093/treephys/tpu076

CHAVE, J.; COOMES, D.; JANSEN, S.; LEWIS, S. L.; SWENSON, N. G.; ZANNE, A. E.. Towards a worldwide wood economics spectrum. Ecology Letters, v.12, n.1, p.351-366. 2009. DOI: https://doi.org/10.1111/j.1461-0248.2009.01285.x

EVANGELISTA, W. V.; SILVA, J. C.; VALLE, M. L. A.; XAVIER, B. A.. Caracterização anatômica quantitativa da madeira de clones de Eucalyptus camaldulensis Dehnh. e Eucalyptus urophylla S. T. Blake. Scientia Forestalis, Piracicaba, v.38, n.86, p.273-284, 2010.

FPL. Forest Products Laboratory. Wood Handbook, Wood as an Engineering Material. Madison: U.S. Department of Agriculture, 2010.

FRANKLIN, G. L.. Preparation of thin sections of synthetic resin and wood: resin composites, and a new macerating method for wood. Nature, London, v.155, n.3924, p.5, 1945. DOI: https://doi.org/10.1038/155051a0

KIM, Y. S.; FUNADA, R.; SING, A. P.. Secondary Xylem Biology: Origins, Functions, and Applications. Massachusett: Academic Press, 2016.

LEITE, G. L. D.; VELOSO, R. V. S.; ZANUNCIO, J. C.; FERNANDES, L. A.; ALMEIDA, C. I. M.. Phenology of Caryocar brasiliense in the Brazilian cerrado region. Forest Ecology and Management, v.236, n.1, p.286-294, 2006. DOI: https://doi.org/10.1016/j.foreco.2006.09.013

LONGUI, E. L.; LIMA, I. L.; SILVA JÚNIOR, F. G.; BUFOLO, A.; SUCKOW, I. M. S.; FLORSHEIM, S. M. B.. Variação radial das características anatômicas, densidade aparente, teores de extrativos, lignina e holocelulose na madeira de Piptadenia gonoacantha (Mart.) Macbr. Scientia Forestalis, v.38, n.87, p.341-353, 2010

MAURI, R.; ALVES, J. A.; OLIVEIRA, J. T. S.; LIMA, J. T.; TOMAZELLO FILHO, M.; CALEGARIO, N.. Morfologia das fibras do lenho de clones de Eucalyptus urophylla $\mathrm{x}$ Eucalyptus grandis em diferentes topografias e altitudes. Scientia Forestalis, v.45, n.113, p.77-87, 2017. DOI: https://doi.org/10.18671/scifor.v45n113.07

MEINZER, F. C.. Functional convergence in plants responses to the environment. Oecologia, v.134, n.1, p.1-11, 2003. DOI: https://doi.org/10.1007/s00442-002-1088-0

MELO, R. R.; DEL MENEZZI, C. H.; SOUZA, M. R.; STANGERLIN, D. M.. Evaluation of physical, chemical, mechanical and surface properties of the Schizolobium amazonicum veneer. Floresta e Ambiente, v.20, n.12, p.238-249, 2013. DOI: https://doi.org/10.4322/floram.2013.00

MOURA, N. F.; CHAVES, L. J.; NAVES, R. V.; AGUIAR, A. V.; SOBIERAJSKI, G. R.. Variability among provenances and progenies of Caryocar brasiliense Camb. Scientia Forestalis, v.41, n.97, p.103-112, 2013.
MULLER-LANDAU, H. C.. Interspecific and inter-site variation in wood specific gravity of tropical trees. Biotropica, v.36, n.1, p.20-32, 2004. DOI: https://doi.org/10.1111/j.17447429.2004.tb00292.x

PALHARES, D.; FRANCO, A. C.; ZAIDAN, L. B. P.. Respostas fotossintéticas de plantas de cerrado nas estações seca e chuvosa. Revista Brasileira de Biociências, v.8, n.2, p.213220, 2010.

PANSHIN, A. J.; ZEEUW, C.. Textbook of wood technology. 3 ed. New York: McGraw Hill, 1980.

PAROLIN, P.. Radial gradients in wood specific gravity in trees of central Amazonian floodplains. IAWA Journal, v.23, n.4, p.449-457, 2002. DOI: https://doi.org/10.1163/22941932-90000314

R CORE TEAM. R: A language and environment for statistica computing. Vienna: R Foundation for Statistical Computing, 2017.

RAMOS, L. M. A.; LATORRACA, J. V. F.; PASTRO, M. S.; SOUZA, M. T.; GARCIA, R. A.; CARVALHO, A. M.. Variação radial dos caracteres anatômicos da madeira de Eucalyptus grandis W. Hill Ex Maiden e idade de transição entre lenho juvenil e adulto. Scientia Forestalis, v.39, n.92, p.411-418, 2011.

RAVEN, P. H.; EICHHORN, S.; EVERT, R. F.. Biologia Vegetal. 8 ed. São Paulo: Guanabara Koogan, 2014

RODRIGUEZ-ZACCARO, F. D.; GROOVER, A.. Wood and water: How trees modify wood development to cope with drought. Plants, People, Planet, v.1, n.4, p.346-355, 2019. DOI: https://doi.org/10.1002/ppp3.29

SANTOS, H. G.; JACOMINE, P. K.; ANJOS, L. H. C.; OLIVEIRA, V. A.; LUMBRERAS, J. F.; COELHO, M. R.; ALMEIDA, J. A.; ARAÚJO FILHO, J. C.; OLIVEIRA, J. B.; CUNHA, T. J. F.. Sistema brasileiro de classificação de solos. Brasília: Embrapa, 2018

SILVA JÚNIOR, M. C.. 100 Árvores do cerrado: sentido restrito: guia de campo. Brasília: Rede de Sementes do Cerrado, 2012.

VALENTE, B. M. R. T.; EVANGELISTA, W. V.; SILVA, J. C. LUCIA, R. M. D.. Variabilidade radial e longitudinal das propriedades físicas e anatômicas da madeira de angicovermelho. Scientia Forestalis, v.41, n.100, p.485-496, 2013.

VOIGT, A. R. A.; SOFFIATTI, P.; TOMAZELLO FILHO, M.; LISI, C. S.; BOEGER, M. R. T.. Anatomia comparada do lenho de Caryocar brasiliense (Caryocaraceae) em fitofisionomias de cerradão e cerrado sensu stricto. Rodriguésia, v.61, n.4, p.603-613, 2010.DOI: https://doi.org/10.1590/21757860201061404

WILLIAMSON, G. B.; WIEMANN, M. C.. Age versus size determination of radial variation in wood specific gravity: lessons from eccentrics. Trees, v.25, n.4, p.585-591, 2011. DOI: https://doi.org/10.1007/s00468-010-0535-5

A CBPC - Companhia Brasileira de Produção Científica (CNPJ: 11.221.422/0001-03) detém os direitos materiais desta publicação. Os direitos referem-se à publicação do trabalho em qualquer parte do mundo, incluindo os direitos às renovações, expansões e disseminações da contribuição, bem como outros direitos subsidiários. Todos os trabalhos publicados eletronicamente poderão posteriormente ser publicados em coletâneas impressas sob coordenação da Sustenere Publishing, da Companhia Brasileira de Produção Científica e seus parceiros autorizados. Os (as) autores (as) preservam os direitos autorais, mas não têm permissão para a publicação da contribuição em outro meio, impresso ou digital, em português ou em tradução. 